\title{
Role of Coelioscopy in the Etiologic Diagnosis of Exudative Ascites of Unknown Origin of the Women
}

\author{
Mohamed Ben Mabrouk ${ }^{*}$, Aida Ben Slama Trabelsi², Mohamed Ben Rejeb ${ }^{3}$, Hanène Jaziri², \\ Mehdi Ksiaa², Sassi Bouguizane4, Badreddine Sriha' ${ }^{5}$, Ali Jmaa' ${ }^{2}$ Ali Ben Ali1 \\ ${ }^{1}$ Department of Digestive Surgery, Sahloul Hospital, Sousse, Tunisia \\ ${ }^{2}$ Department of Gastroenterology, Sahloul Hospital, Sousse, Tunisia \\ ${ }^{3}$ Department of Preventive Medicine, Sahloul Hospital, Sousse, Tunisia \\ ${ }^{4}$ Department of Gynecology, Farhat Hached Hospital, Sousse, Tunisia \\ ${ }^{5}$ Department of Pathology, Farhat Hached Hospital, Sousse, Tunisia \\ Email: ${ }^{*}$ mohamed.benmabrouk@rns.tn
}

Received 8 February 2015; accepted 23 May 2015; published 27 May 2015

Copyright (C) 2015 by authors and Scientific Research Publishing Inc.

This work is licensed under the Creative Commons Attribution International License (CC BY).

http://creativecommons.org/licenses/by/4.0/

(c) (7) Open Access

\begin{abstract}
Introduction: The aim of this study was to clarify the role of laparoscopy in the etiological diagnosis of exudative ascites of unknown origin in women. Materials and Methods: This was a prospective study from 2007 to 2012, including 83 women with exudative ascites of unknown origin and had a laparoscopy diagnostic purpose. Results: Carcinomatosis and peritoneal tuberculosis were the two most common causes found in $31 \%$ (26 cases) and $66 \%$ (55 cases) of cases respectively. The average age of the patients was 56 years. Weight loss and abdominal pain were the most frequent, observed symptoms in 71 cases $(85.5 \%)$ and 53 cases $(63.8 \%)$ respectively. The CT scan abnormalities noted were a peritoneal thickening in 32 cases $(38.5 \%)$, peritoneal nodules in 15 cases (18\%), and agglutination of the digestive handles in 12 cases (14.4\%). Laparoscopic Visual diagnosis was in favor of a peritoneal carcinomatosis in 32 cases $(38.5 \%)$, peritoneal tuberculosis in 45 cases $(54.2 \%)$, and non specific infection in 6 cases $(7.3 \%)$. The histological diagnosis was a peritoneal carcinomatosis in 26 cases $(31 \%)$, and peritoneal tuberculosis in 55 cases $(66 \%)$. The positive predictive value of laparoscopic vision Diagnostics in Peritoneal tuberculosis was $\mathbf{1 0 0 \%}$ and the negative predictive value was $73.7 \%$. In peritoneal carcinomatosis, the positive predictive value was $81.3 \%$ and the negative predictive value was $100 \%$. Conclusion: The etiologic diagnosis of exudative ascites in women is difficult despite the availability of several tests. Currently, laparoscopy with peritoneal biopsy remains the gold standard for etiologic diagnosis.
\end{abstract}

${ }^{*}$ Corresponding author. 


\section{Keywords}

\section{Ascites, Exudative, Laparoscopy, Peritoneal Tuberculosis, Peritoneal Carcinomatosis}

\section{Introduction}

Exudative ascites is a frequent clinical entity that can be secondary to several pathologies. The usual diagnostic approach before ascites contains, in addition to physical examination, biological examinations (blood and ascites) and radiological examinations that cannot identify the cause of ascites in the majority of cases. The etiologies of these ascites, say of unknown origin, are dominated by tuberculosis and peritoneal carcinomatosis requiring diagnosis and early care [1] [2]. Thus, biopsies peritoneal per laparoscopic are required for etiological diagnosis.

The aim of this work was to describe the epidemiological, clinical, morphological, and macroscopic aspects of tuberculosis and peritoneal carcinomatosis and clarify the predictive value of the laparoscopic Visual diagnosis and histology in etiological diagnosis of exudative ascites of unknown origin in women.

\section{Materials and Methods}

\subsection{Type of the Study}

It is a prospective study including all the women who have been explored for exudative ascites in Gastroenterology Department of Sahloul Hospital in Sousse and who had a diagnostic laparoscopy used during the period from January 2007 to December 2012.

\subsection{Criteria for Inclusion}

During the period of the study, 83 patients were explored for exudative ascites. Before doing diagnostic laparoscopy, all women have had an exhaustive exploration with: an examination with a complete physical examination, tuberculosis review (intradermoreaction to tuberculin, chest x-ray, search for Koch's Bacillus in sputum, urine and ascites), a paracentesis of ascites fluid Explorer (cellularity, formula, direct examination with search of germs, cytology with search of neoplastic cells, culture of ascitic fluid, proteins), biological blood tests (blood count blood and tumor markers), endoscopic examinations (high digestive fibroscopy, colonoscopy) and radiological tests (ultrasound transvaginal, thoraco-abdomino-pelvic scanner). When this survey came back negative, the patient was clerk for diagnostic laparoscopy.

\subsection{Technique}

During exploration, ascitic fluid aspiration was performed, and clarified each macroscopic anomaly with details (nature, size, number, color, seat...). Multiple biopsies were systematically conducted during the review.

The Visual diagnosis of Peritoneal tuberculosis was suspected before the presence of multiple small nodules or granules $(<5 \mathrm{~mm})$ of uniform size, white or yellowish, and scattered on the parietal, visceral peritoneum and viscera.

Peritoneal carcinomatosis was suspected before the presence of nodules of larger size (1 - $5 \mathrm{~cm}$ in diameter) on the peritoneum and viscera.

The histological diagnosis of peritoneal tuberculosis has been selected before the presence of giant cell granulomas with or without caseous necrosis.

\subsection{Statistical Analysis and Expression of Results}

The sensitivity, specificity, predictive value positive and negative of laparoscopy are calculated. The data entry as well as the statistical analysis is performed using SPSS version 11.0 software.

\section{Results}

Over a period of 6 years, we collected 83 patients meet the criteria for inclusion. Carcinomatosis and peritoneal 
tuberculosis were the two most common causes found in 31\% (26 cases) and 66\% (55 cases) of cases respectively. The average age of patients was $56 \pm 17$ years with extremes from 17 to 74 years.

Weight loss and abdominal pain were the most frequent, observed symptoms in 71 cases (85.5\%) and 53 cases (63.8\%) respectively. The signs of tuberculosis impregnation were significantly more frequent in peritoneal tuberculosis $(\mathrm{P}<0.01)$.

Ascites was the reason for consultation of all patients. The appearance of ascites fluid was yellow citrine in 61 cases (73.5\%) and hemorrhagic in 22 cases (26.5\%). Ascites was lymphocyte-rich (>1000 elmt/mm $\left.{ }^{3}\right)$ in 39 cases (47\%). Direct examination and research of Koch's Bacillus and the culture of the ascites were negative in all cases.

A biology, the sedimentation speed (VS) was accelerated in 76 cases (91.5\%) and the rate of CA - 125 was raised in 74 cases (89\%).the anomalies noted on CT scan, were a peritoneal thickening in 32 cases (38.5\%), peritoneal nodules in 15 cases (18\%), agglutination of the digestive handles in 12 cases (14.4\%), and the intraabdominal lymph nodes in 17 cases (20.5\%). An ovarian mass was found in 12 cases (14.4\%).

The main clinical, biological and morphological elements are summarized in Table 1 on the basis of two main etiologies.

Clinical signs, biological explorations and morphological examinations were not contributing to the etiologic diagnosis. All patients had benefited of a diagnostic laparoscopy.

Laparoscopic elementary lesions were peritoneal nodules in 26 cases (31.3\%), and peritoneal granules in 41 cases (49.4\%). Other associated lesions were found: adhesions in 59 cases (71\%), peritoneal hyperaemia in 48 cases (57\%), and agglutination of the digestive handles in 20 cases (24\%).

Only adhesions without nodules or granules were present in 8 cases $(9.6 \%)$. The histological diagnosis in these forms was a peritoneal tuberculosis in 6 cases, a non-specific inflammatory reworking in one case and a chlamydia infection in another case (Table 2).

Laparoscopic Visual diagnosis was in favour of a peritoneal carcinomatosis in 32 cases (38.5\%), peritoneal tuberculosis in 45 cases (54.2\%), and nonspecific infection in 6 cases (7.3\%).

Table 1. Clinico-biological and CT features of patients according to the two main etiologies.

\begin{tabular}{lcc}
\hline & $\begin{array}{c}\text { Peritoneal tuberculosis } \\
\mathrm{N}=55\end{array}$ & $\begin{array}{c}\text { Peritoneal carcinomatosis } \\
\mathrm{N}=26\end{array}$ \\
\hline Abdominal pain & $37(67 \%)$ & $16(61 \%)$ \\
Weight loss & $49(89 \%)$ & $22(84.6 \%)$ \\
Fever and night sweats & $33(60 \%)$ & $4(15 \%)$ \\
Ascitic fluid & & \\
- Citrus yellow & $52(94.5 \%)$ & $9(34.6 \%)$ \\
- Haemorrhagic & $4(7 \%)$ & $18(69 \%)$ \\
- Average lymphocytes & 1243 & 512 \\
Sedimentation rate & $52(94 \%)$ & $24(92 \%)$ \\
Increased CA-125 & $50(91 \%)$ & $24(92 \%)$ \\
CA-125 $\geq 20 \mathrm{~N}$ & $5(9 \%)$ & $15(57 \%)$ \\
Impairing aspects & & $12(46 \%)$ \\
- Peritoneal thickening & $20(36 \%)$ & $13(50 \%)$ \\
- Peritoneal nodules & $2(3.5 \%)$ & $1(4 \%)$ \\
- Agglutination of the handles & $11(20 \%)$ & $7(26 \%)$ \\
- Abdominal adenopathy & $11(20 \%)$ &
\end{tabular}


The histological diagnosis was a peritoneal carcinomatosis of ovarian origin in 26 cases (31.3\%), peritoneal tuberculosis in 55 cases (66.2\%), infection with chlamydia (Fitz-Hugh-curtis syndrome) in one case and nonspecific inflammation in one case.

Giant cell granulomas were present in all patients with peritoneal tuberculosis. Caseous necrosis was found in half of the cases.

In all cases, peritoneal carcinomatosis was of ovarian origin.

The correlation between Visual laparoscopic diagnosis and histology is represented in Table 3.

The study of the correlation between Visual laparoscopic diagnosis and histology allowed us to estimate a coefficient of concordance 0.752 kappa $+/-0.071(\mathrm{P}<0.01)$.

The sensitivity and specificity of the laparoscopic Visual diagnosis in the diagnosis of peritoneal tuberculosis were $81.8 \%$ and $100 \%$ respectively. The positive predictive value was $100 \%$ and the negative predictive value was $73.7 \%$.

The sensitivity and specificity of the laparoscopic Visual diagnosis in the diagnosis of peritoneal carcinomatosis were $100 \%$ and $89.5 \%$ respectively. The positive predictive value was $81.3 \%$ and the negative predictive value was $100 \%$ (Table 4$)$.

The length of postoperative stay was $36 \pm 12$ hours. A single per-operative complication was observed in our series. This complication was type of active bleeding a peritoneal biopsy. Neoplasic spread on the trocar site was occurred in one case three months after the diagnostic laparoscopy.

Table 2. The laparoscopic elementary lesions according to the two main etiologies.

\begin{tabular}{ccc}
\hline & Peritoneal tuberculosis & Peritoneal carcinomatosis \\
\hline Peritoneal nodules & 0 & $26(100 \%)$ \\
Peritoneal granules & $48(87 \%)$ & 0 \\
Adhesions & $40(72.7 \%)$ & $19(73 \%)$ \\
Only adhesions & $6(11 \%)$ & 0 \\
Peritoneal hyperemia & $32(58 \%)$ & $16(61.5 \%)$ \\
Agglutination of the digestive handles & $20(36 \%)$ & 0 \\
\hline
\end{tabular}

Table 3. Crosswalk between laparoscopic Visual diagnosis and histology.

\begin{tabular}{cccccc}
\hline & & \multicolumn{2}{c}{ Laparoscopic vision Diagnostics } & Total \\
\cline { 3 - 5 } & & $\begin{array}{c}\text { Peritoneal } \\
\text { tuberculosis }\end{array}$ & $\begin{array}{c}\text { Peritoneal } \\
\text { carcinomatosis }\end{array}$ & Other & \\
\hline \multirow{2}{*}{$\begin{array}{c}\text { Histological } \\
\text { diagnosis }\end{array}$} & Peritoneal tuberculosis & 45 & 6 & 4 & 55 \\
& Other & 0 & 26 & 2 & 2 \\
\hline
\end{tabular}

Table 4. Sensitivity and specificity of the laparoscopic Visual diagnosis in tuberculosis and peritoneal carcinomatosis.

\begin{tabular}{ccccc}
\hline & SE & SP & VPP & VPN \\
\hline Tuberculosis & $81.8 \%[68-90]$ & $100 \%[85-100]$ & $100 \%[90-100]$ & $73.7 \%[56-86]$ \\
Carcinomatosis & $100 \%[84-100]$ & $89.5 \%[77-95]$ & $81.3 \%[63-92]$ & $100 \%[91-100]$ \\
\hline
\end{tabular}

SE: sensitivity; SP: specificity; VPP: positive predictive value; VPN: negative predictive value; []: 95\% confidence interval. 


\section{Discussion}

Exudative ascites of unknown origin in women are dominated by tuberculosis and peritoneal carcinomatosis requiring diagnosis and early care [1] [2]. The means of imaging (ultrasound, CT scan, MRI) have limited coverage in the etiological diagnosis of exudative ascites [3] [4].

Several series of literature [5]-[11], confirmed the feasibility of laparoscopy in the exploration of the ascites of unknown origin in women as well as its high sensitivity and specificity. The main advantage of laparoscopy compared with other explorations through optical magnification, it allows an excellent exploration of peritoneal surfaces and the abdominopelvic cavity [12]. Biopsies are taken under direct control of the view, contrary to those obtained by imaging. Therefore, laparoscopy enables to distinguish between peritoneal tuberculosis and a carcinomatosis peritoneal [13].

During tuberculosis, three lesions have been described, most commonly associated in the same patient. Peritoneal granulations are color whitish or yellowish, uniform size, the size of a pin head, not exceeding $5 \mathrm{~mm}$, divided equally as well at the level of the parietal peritoneum that visceral. Adhesions result from the Organization of fibrinous exudates, between two peritoneal layers. These adhesions can have an aspect cobweb son or a thicker appearance pillars or ropes [14]. The inflammatory phenomena manifested by congestion, hypervascularisation and edematous state of peritoneum [15].

In literature, the grits are the most frequently encountered aspect (66\% to $100 \%$ of the cases), adhesions were observed in $13 \%$ to $80 \%$ of cases, and inflammatory phenomena are described in $21 \%$ to $79 \%$ of the cases [14] [16]-[19].

For our patients with peritoneal tuberculosis, the grits were observed in $87 \%$ of cases, adhesions in $72 \%$ cases and inflammatory phenomena in $58 \%$ of cases.

In the peritoneal carcinomatosis, peritoneal implants are generally size different, upper to $1 \mathrm{~cm}$ and distributed irregularly on the peritoneum, abdomino-pelvic viscera and diaphragm [13]. In general, multiple biopsies at the primary tumor if it was identified and peritoneal implants are needed. Apart from his diagnostic interest, laparoscopy to predict the tumor resectability in cases of advanced ovarian cancer and thus avoiding laparotomy and unnecessary surgery as part of an already disseminated tumor.

In the series of Barnard et al. [2] involving 90 patients, including 60 women, the positive predictive value of the laparoscopy in peritoneal tuberculosis was $85 \%$. Peritoneal biopsy confirmed the diagnosis of tuberculosis and peritoneal carcinomatosis in $98 \%$ and $100 \%$ of the cases respectively.

Chien-Min Han et al. [6] investigated the sensitivity and specificity of Visual diagnostic laparoscopic in 176 patients with ascites transudative and exudative of undetermined origin. The sensitivity and specificity of diagnostic Visual laparoscopic peritoneal tuberculosis were $86 \%$ and $100 \%$ respectively. In the peritoneal carcinomatosis, the sensitivity and specificity were $100 \%$ and $94 \%$, respectively.

Sheth SS et al. [5] assessed the contribution of laparoscopy in ascites of unknown origin in 70 women. Laparoscopy and peritoneal biopsies have highlighted the diagnosis in $90 \%$ of cases.

Nassir et al. [8] assessed the contribution of laparoscopy in exuding ascites of unknown origin in 33 patients whose 18 women. The diagnostic profitability of this exploration was $97 \%$.

In our series, sensitivity and specificity of the laparoscopic Visual diagnosis in the diagnosis of peritoneal tuberculosis were $81.8 \%$ and $100 \%$ respectively. In the peritoneal carcinomatosis, the sensitivity and specificity were $100 \%$ and $89.5 \%$ respectively. This shows that the only Visual laparoscopic diagnosis helps to confirm the diagnosis of tuberculosis and eliminate a carcinomatosis in $100 \%$ of cases. Histology allowed to confirm the diagnosis of tuberculosis and peritoneal carcinomatosis in all cases.

\section{Conclusion}

The etiological diagnosis of exudative ascites in women is difficult despite the availability of several tests expensive and complex. Currently, laparoscopy with peritoneal biopsy remains the gold standard for etiologic diagnosis.

\section{References}

[1] Hasson, H.M. (1974) Open Laparoscopy: A Report of 150 Cases. Journal of Reproductive Medicine, 12, 234-238.

[2] Bedoui, H., Ksantini, R., Nouira, K., et al. (2007) Role of Laparoscopic Surgery in the Etiologic Diagnosis of Ex- 
sudative Ascites: A Prospective Study of 90 Cases. Gastroentérologie Clinique et Biologique, 31, 1146-1149. http://dx.doi.org/10.1016/S0399-8320(07)78354-9

[3] Boughizane, S., Chaieb, A., Hidar, S., Darraji, F., Lassaouad, L. and Khairi, H. (2003) Introduction du trocart initial au niveau de l'hypochondre gauche en gynécologie. A propos d’une série de 45 cas. Le Journal de Colio-Chirurgie, 47, 65-69.

[4] Parsons, S.L., Lang, M.W. and Steele, R.J.C. (1996) Malignant Ascites: A 2-Year Review from a Teaching Hospital. European Journal of Surgical Oncology, 22, 237-239. http://dx.doi.org/10.1016/S0399-8320(07)78354-9

[5] Sheth, S.S. (1989) The Place of Laparoscopy in Women with Ascites. British Journal of Obstetrics and Gynaecology, 96, 105-106. http://dx.doi.org/10.1111/j.1471-0528.1989.tb01585.x

[6] Han, C.M., Lee, C.L., Huang, K.G., et al. (2008) Diagnostic Laparoscopy in Ascites of Unknown Origin: Chang Gung Memorial Hospital 20 Year Experience. Chang Gung Medical Journal, 31, 378-383.

[7] Milingos, S., Protopapas, A., Papadimitriou, C., et al. (2007) Laparoscopy in the Evaluation of Women With Unexplained Ascites: An Invaluable Diagnostic Tool. Journal of Minimally Invasive Gynecology, 14, 43-48. http://dx.doi.org/10.1016/j.jmig.2006.06.020

[8] Luck, N.H., Khan, A.A., Alam, A., Butt, A.K. and Shafquat, F. (2007) Role of Laparoscopy in the Diagnosis of Low Serum Ascites Albumin Gradient. Journal of Pakistan Medical Association, 57, 33-34.

[9] Chow, K.M., Chow, V.C.Y. and Szeto, C.C. (2003) Indication for Peritoneal Biopsy in Tuberculous Peritonitis. The American Journal of Surgery, 185, 567-573. http://dx.doi.org/10.1016/S0002-9610(03)00079-5

[10] Bhargava, D.K., Shriniwas, Chopra, P., Nijhawan, S., Dasarathy, S. and Kushwaha, A.K. (1992) Peritoneal Tuberculosis: Laparoscopic Patterns and Its Diagnostic Accuracy. The American Journal of Gastroenterology, 87, $109-112$.

[11] Sharma, M.P. and Bhatia, V. (2004) Abdominal Tuberculosis. Indian Journal of Medical Research, 120, 305-315

[12] Henning, H. (1992) Indications and Contraindications of Diagnostic Laparoscopy. Endoscopy, 24, 674-675. http://dx.doi.org/10.1055/s-2007-1010559

[13] Orlando, R., Chiarion, C. and Figlioli, G.F. (1996) La laparoscopie est-elle encore utile dans l'évaluation des ascites. Acta Endoscopica, 26, 159-162. http://dx.doi.org/10.1007/BF02965804

[14] Singh, M.M., Bhargava, A.N. and Jain, K.P. (1969) Tuberculous Peritonitis. The New England Journal of Medicine, 281, 1091-1094. http://dx.doi.org/10.1056/NEJM196911132812003

[15] Etienne, J.P., Chretien, J. and Christoforov, B. (1966) Les péritonites tuberculeuses de l’adulte (à propos de 35 observations). Sem Hop Ther Paris, 42, 813-820.

[16] Bennani, A., Ouazzani, H., Fadili, F., Dafiri, N. and Ouazzani, L. (1988) Diagnostic et aspects thérapeutiques de la tuberculose péritonéale au Maroc. A propos de 300 cas. The Annals of Gastroenterology \& Hepatology, 24, 347-354.

[17] El Ajmi, S., Chatti, N. and Limam, K. (1991) La tuberculose péritonéale, aspects actuels à propos de 39 cas observés au centre Tunisien. Médecine du Maghreb, 27, 11-12.

[18] Hamdani, A., Sekkat, N., Alyoune, A., et al. (1987) Tuberculose péritonéale chez l'adulte. Etude de 207 cas. The Annals of Gastroenterology \& Hepatology, 3, 115-122.

[19] Redah, D., Amedegnato, D., Napo-Koura, G, et al. (1993) La tuberculose péritonéale au CHU de Lombé-Tokion à propos de 108 cas. Médecine \& Chirurgie Digestives, 22, 105-106. 